$$
\begin{aligned}
& \text { 震 }
\end{aligned}
$$

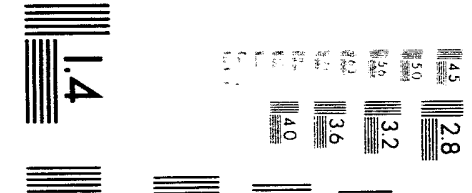

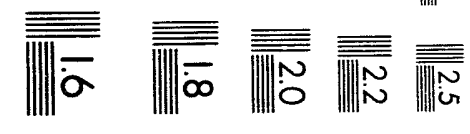



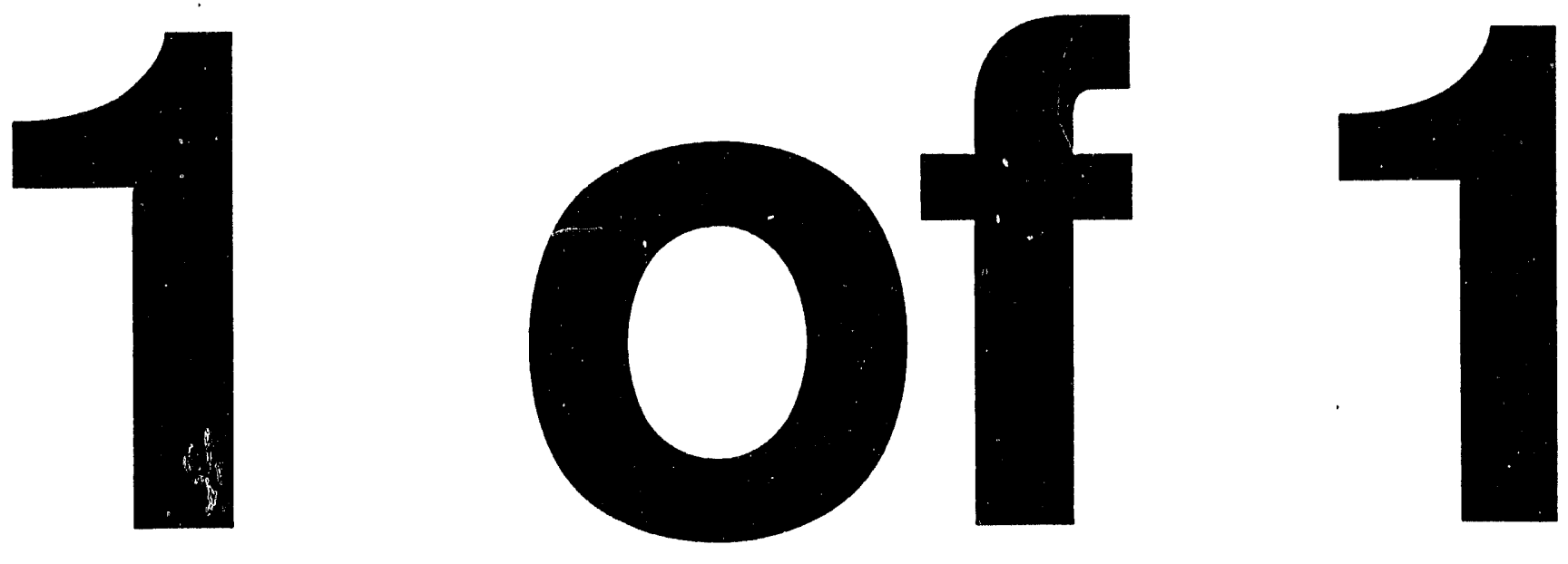


\title{
INDIUM DONOR COMPLEXES WITH CATION VACANCIES IN CdTe AND ZnSe*
}

\author{
J. W. GRIFFITH*, R. LUNDQUIST*, R. PLATZER*, JOHN A. GARDNER*, G. \\ KARCZEWSKI ${ }^{\dagger}$ and J. K. FURDYNA ${ }^{\dagger}$
}

* Department of Physics, Oregon State University, Corvallis, Oregon 97331

'Department of Physics, University of Notre Dame, Notre Dame, IN 46556

\begin{abstract}
Very dilute $\left(\sim 10^{12} \mathrm{~cm}^{-3}\right)$ indium donors in CdTe and $\mathrm{ZnSe}$ powders and in CdTe single crystals have been investigated using ${ }^{111}$ In Perturbed angular correlation spectroscopy. Most indium atoms are in uncomplexed sites but can form weakly-bound complexes with native defects in very defective material. The only complex observed in CdTe is an indium-Cd vacancy pair. The CdTe in which these pairs occur is apparently n-type, most $\mathrm{Cd}$ vacancies are free and doubly-charged, and the binding energy with indium is $0.15 \mathrm{eV}$. In $\mathrm{ZnSe}$, indium can pair with a $\mathrm{Zn}$ vacancy or with some other presently unidentified defect. These complexes form in ZnSe containing large concentrations of both free $\mathrm{Zn}$ vacancies and complexes of $\mathrm{Zn}$ vacancies with other defects. In CdTe, the pair formation equilibration time constant is two days at $15^{\circ} \mathrm{C}$, an implication that $\mathrm{Cd}$ vacancies are mobile at room temperature. Lattice relaxation around a $\mathrm{Cd}$ vacancy in $\mathrm{CdTe}$ was probed by single crystal PAC experiments.
\end{abstract}

In this study, ${ }^{111} \mathrm{in} / \mathrm{Cd}$ perturbed angular correlation (PAC) spectroscopy[1,2] was used to detect complexes with dilute radioactive indium dopants. CdTe (ZnSe) Powder samples were annealed at $800(1100)^{\circ} \mathrm{C}$ to diffuse in approximately $10^{11} 111 \mathrm{In}$ atoms homogeneously and introduce large native defect concentrations. PAC spectra of several CdTe single crystal samples were also measured. These samples were made in a qualitatively similar manner to the powders, but were annealed at only $550^{\circ} \mathrm{C}$ to introduce the indium and native defects. Higher temperatures caused the single crystals to become polycrystalline.

PAC measurements were done using a standard 4-detector PAC spectrometer. [2] Fig. 1 shows room temperature single crystal CdTe PAC time spectra and the corresponding Fourier transforms for the three cases - detectors aligned with the $\langle 100\rangle,\langle 110\rangle$ and $\langle 111\rangle$ crystal axes. In these spectra, approximately half the indium atoms are uncomplexed, and the remainder are subject to interaction with a bound $\mathrm{Cd}$ vacancy. [3] From the orientation-dependence of the intensity of the three PAC frequency components, one can determine the orientation of the efg caused by the bound $\mathrm{Cd}$ vacancy.

*This research was supported in part by the U.S. Department of Energy.

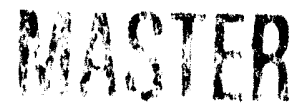


In the absence of lattice relaxation, the efg would be axially symmetric and oriented in a $\langle 110\rangle$ direction. We find experimentally an asymmetry of 0.16 and a $V_{2 z}$ orientation closer to $\langle 111\rangle$. The efg principal axis $V_{z z}$ is directed at an angle of roughly 55 degrees with respect to the $\langle 110\rangle$ direction. Additional data are required to improve the accuracy of this determination. Lattice relaxation, primarily of the Te atom adjacent to both the PAC probe atom and vacancy is almost certainly responsible for departures from the simplest expectations. A more complete description of thie single-crystal work will be published later.

Powder PAC data are qualitatively similar to those shown in Fig. 1. The Fourier transforms of $\mathrm{CdTe}$ and $\mathrm{ZnSe}$ powder PAC data are shown in Fig. 2. Computer fittings of CdTe powder data to a standard PAC model[1,4] show that all indium atoms are uncomplexed or associated with a single $\mathrm{Cd}$ vacancy.

ZnSe PAC data are well-fitted by a three-site model. One site is an indium/Zn vacancy pair. The PAC frequencies for the second complex are smaller, and the efg asymmetry is very large. The asymmetry suggests that a double-vacancy pair or some other larger native defect cluster is bound to the indium. Whenever the complexes are observed, the $G_{2}(t)$ contribution from uncomplexed indium decays slightly with $t$, possibly an indication that the defect density is very large.

PAC frequencies are reproducible from sample to sample. Site fractions depend on temperature and sample preparation conditions. For a given CdTe powder sample site fractions are reproducible on thermal cycling within the temperature range 40 to $200^{\circ} \mathrm{C}$. At much lower temperatures, equilibrium between the indium and quenchedin $\mathrm{Cd}$ vacancies is reached too slowly for useful data to be accumulated. Annealing at higher temperatures can affect the quenched-in native defect concentration in the sample grains. For $\mathrm{ZnSe}$ samples, PAC frequencies are reproducible, but site fractions are not reproducible on thermal cycling.

If the free vacancy concentration $c$ is constant and has the same charge state when free or bound to indium, then the equilibrium ratio of free to complexed indium atoms is given by:

$$
\frac{f_{f}}{f_{c}}=\frac{1}{12 C} \exp ^{-E / k T}
$$

Here $f_{f}$ and $f_{c}$ are respectively the fractions of free and complexed indium, $c$ is the $C d$ vacancy concentration and $E$ is the binding energy of the complexes. An Arrhenius plot of $f_{f} / f_{c}$ for several CdTe powder samples is shown in Fig. 3. Within statistical uncertainty, the slope is the same for all samples, yielding $E=0.15(1) \mathrm{eV}$. Concentrations $c$ are of order $10^{18} \mathrm{~cm}^{-3}$ The concentration $c$ depends on details of sample purity, annealing, and quenching, but the energy $E$ is always the same within statistical uncertainty, a strong indication that the assumption of constant $c$ and charge state are valid for CdTe. 
The $\mathrm{ZnSe}$ site fractions are much more sensitive to preparation conditions. Annealing in $\mathrm{Zn}$ vapor always reduced the pair fractions, but annealing in Se vapor did not always increase them. Many samples had no complexes even after lengthy annealing in Se vapor. Within a temperature range $100-300{ }^{\circ} \mathrm{C}$, spectra were usually reproducible on thermal cycling. The site fraction ratio $f_{4} / f_{c}$ increased with increasing temperature, but Arrhenius plots for neither complex have sample-independent slopes.

We have no explanation for the sample-to-sample nonreproducibility of ZnSe site fraction temperature dependence unless very small concentrations of accidentallyintroduced impurities greatly affect defect equilibrium. Samples were not handled under clean room conditions so we cannot completely exclude interaction with the fused silica container at annealing temperatures, we are confident that accidental contamination is considerably smaller than quenched-in native defect concentrations.

The absence of PAC "aftereffects"[1] and, for ZnSe, the well-known difficulty of pdoping $Z n S e$ imply that these materials are $n$-type, so all free $C d$ and $Z n$ vacancies should be doubly charged acceptors. The observed sample-dependence of Arrhenius slopes of indium $/ \mathrm{Zn}$ vacancy pair fractions imply that the free $\mathrm{Zn}$-vacancy concentration $c$ must be temperature-dependent in at least some samples. $c$ should not depend on temperature unless a substantial fraction of $\mathrm{Zn}$ vacancies are bound into complexes with other native defects. One of these complexes could be the defect associated with the second indium/defect pair.

The site probabilities were generally found to be time dependent for CdTe near room temperature. The pair probability vs. time was measured for one sample quenched to $15^{\circ} \mathrm{C}$ and is shown in Fig. $4 . f_{c}$ approaches an asymptotic value of 0.62 with a time constant of $45(5)$ hours. Vacancy hopping is the dominant diffusion mechanism at high temperature[5], but little is known about transport near room temperature. If vacancy motion is dominant, then these data imply that the $\mathrm{Cd}$ vacancy jump time near room temperature is of order hours. If so, then CdTe devices cannot be stable against $\mathrm{Cd}$ vacancy motion unless cooled well below room temperature. We observed time-dependent effects in $\mathrm{ZnSe}$ but only at temperatures of order $100{ }^{\circ} \mathrm{C}$ or above.

\section{REFERENCES}

[1] R. Wang, J. Gardner, W. Evenson and J. Sommers, Phys. Rev. B 47, 638 (1993).

[2] H. Jaeger, J.A. Gardner, H.T. Su, and R.L. Rasera, Rev. Sci. Instrum. 58, 1697 (1987).

[3] R. Kalish, M. Deicher, and G. Schatz, J. Appl. Phys. 53, 4793 (1982).

[4] J.W. Griffith, R. Lundquist, R. Platzer, and John A. Gardner, submitted for publication, June 1993.

[5] E. Watson and D. Shaw, J. Phys. C 16515 (1983). 
Figure 1. PAC time spectra and Fourier transforms accumulated for CoTe single crystal when detectors are aligned with $(100),\langle 110\rangle$, and (111) directions. For latter case, only 2 detectors are in $(111)$ direction, since detector 90 degree angular spacing is retained.
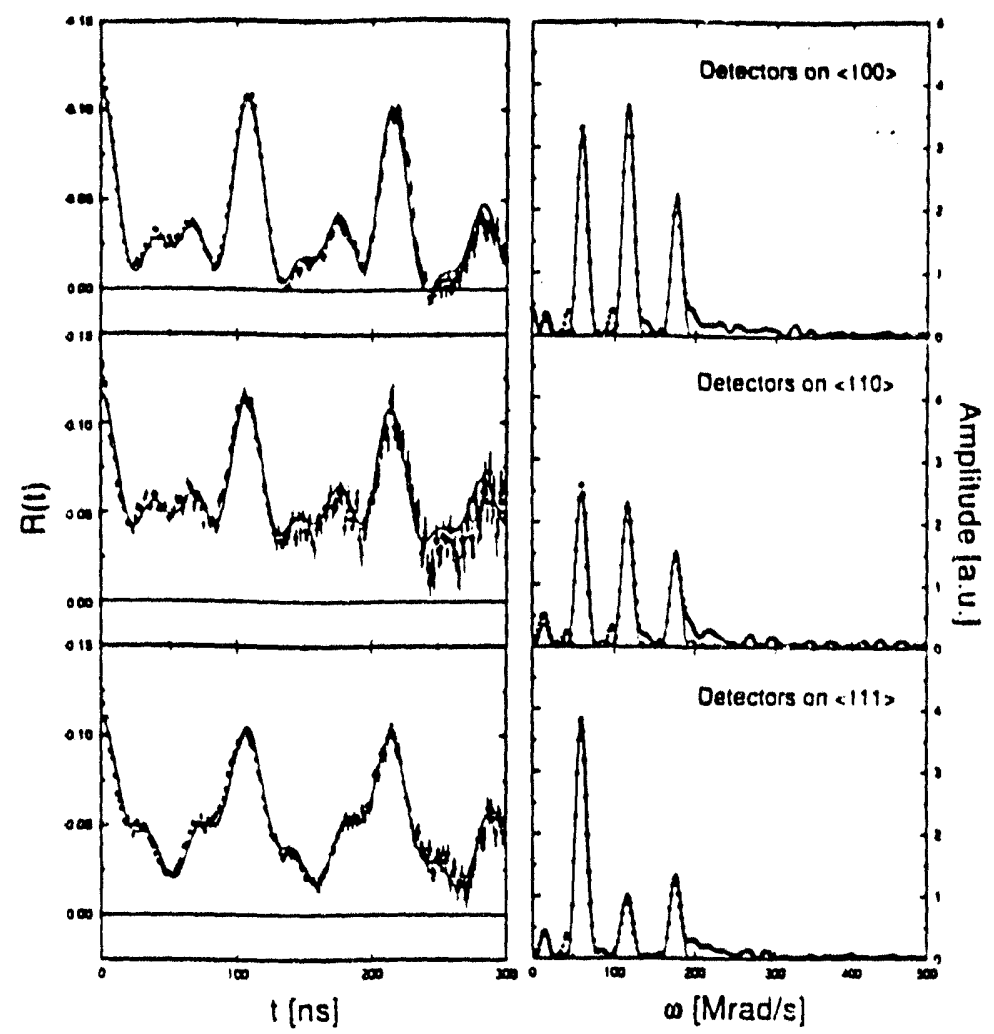

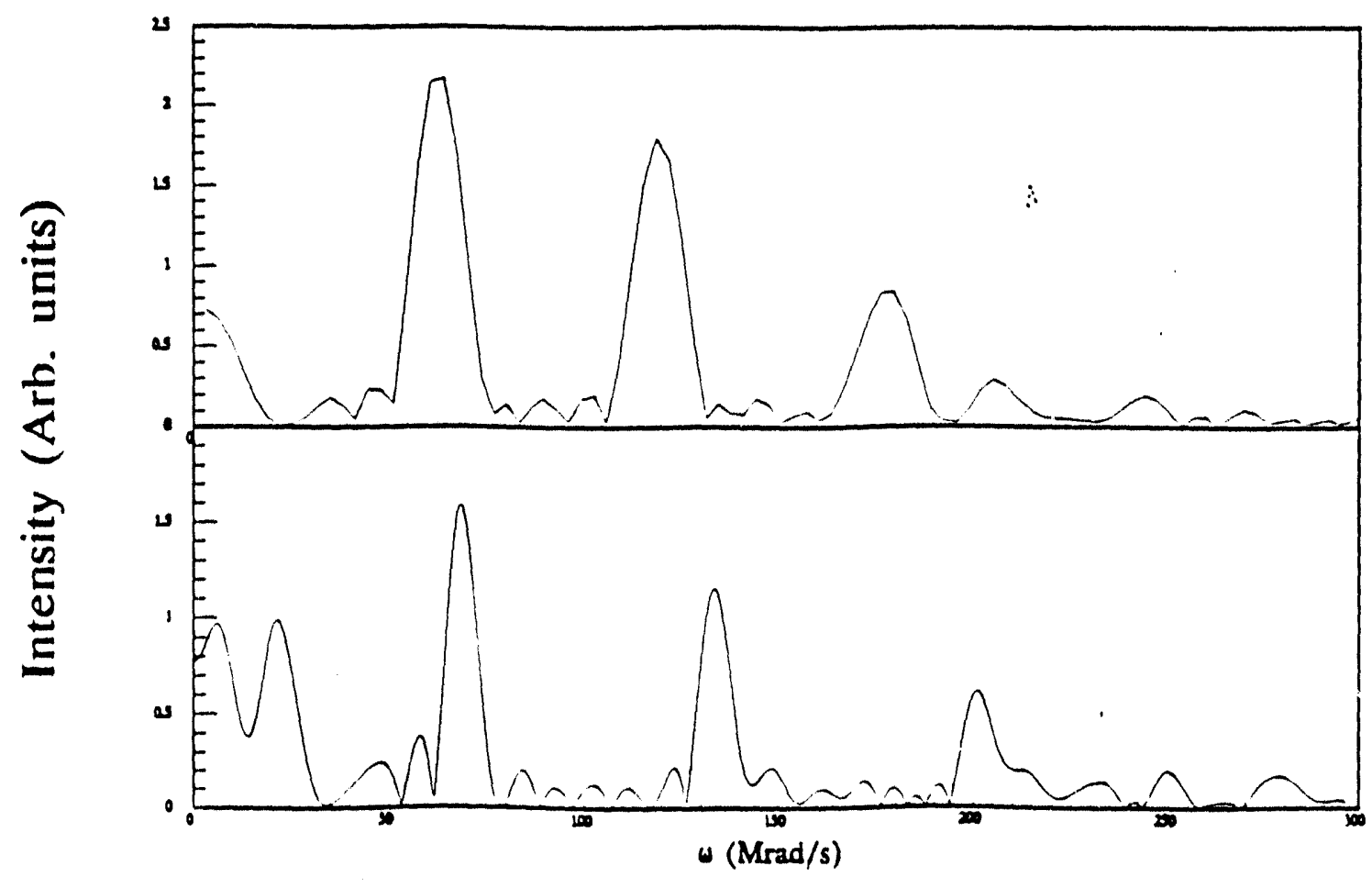

Figure 2. $" 11 \mathrm{n} / \mathrm{Cd}$ PAC fourier transforms. (a) in CdTe at $23^{\circ} \mathrm{C} .65 \%$ of the donors are complexed and subject to static efg interaction. (b) in $\mathrm{ZnSe}$ at $100{ }^{\circ} \mathrm{C}$. $62 \%$ of the donor sites are only marginally perturbed $\left(G_{2}(t)\right.$ decreases slightly with $\left.t\right) ; 25.7 \%$ is subject to a first static efg interaction; and $12.2 \%$ is subject to a second efg interaction. 

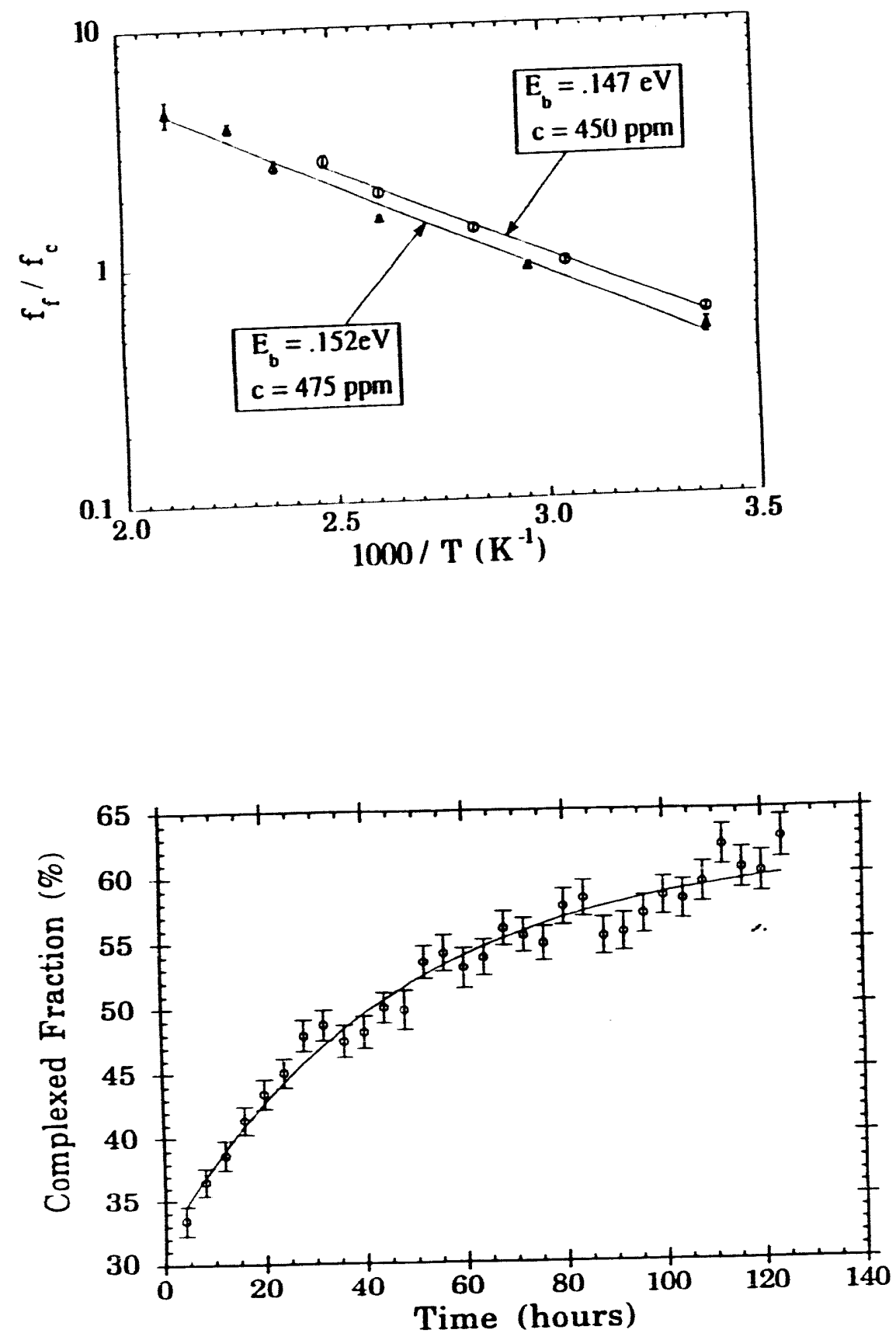

Figure 3. Arrhenius plot of the ratio $f_{1} / f_{c}$ of free to complexed indium dopants. Solid lines are computer fits to Eq. 1.

Figure 4. Fraction $f_{c}$ of indiumvacancy pairs vs. time for a CdTe sample at $15^{\circ} \mathrm{C}$. The sample was annealed with excess $\mathrm{Te}$ at $800^{\circ} \mathrm{C}$ for 5 hours and quenched to room temperature in one minute. 

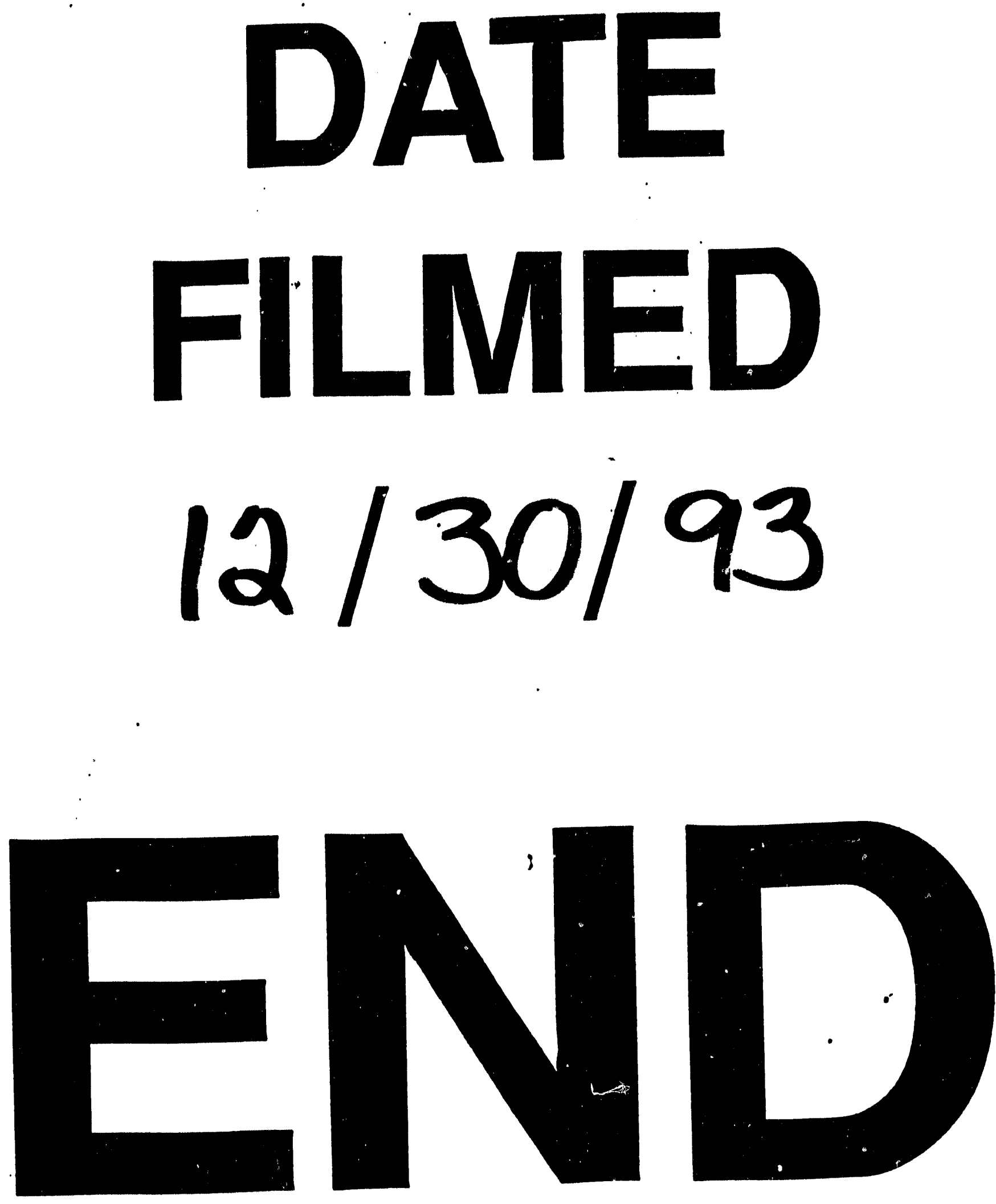
